\title{
Lipophilicity Assessment of Ruthenium(II)-Arene Complexes by the Means of Reversed-Phase Thin-Layer Chromatography and DFT Calculations
}

\author{
Khalil Salem A. M. Shweshein,, ${ }^{1}$ Filip Andrić, ${ }^{1}$ Aleksandra Radoičić, ${ }^{1}$ Matija Zlatar, ${ }^{2}$ \\ Maja Gruden-Pavlović, ${ }^{1}$ Živoslav Tešić, $^{1}$ and Dušanka Milojković-Opsenica ${ }^{1}$ \\ ${ }^{1}$ Faculty of Chemistry, University of Belgrade, Studentski Trg 12-16, P.O. Box 51, 11158 Belgrade, Serbia \\ ${ }^{2}$ Institute for Chemistry, Technology and Metalurgy, Center of Chemistry, University of Belgrade, Njegoševa 12, \\ 11000 Belgrade, Serbia
}

Correspondence should be addressed to Dušanka Milojković-Opsenica; dusankam@chem.bg.ac.rs

Received 29 August 2013; Accepted 28 October 2013; Published 22 January 2014

Academic Editors: C. Platas-Iglesias and S. Sultan

Copyright (C) 2014 Khalil Salem A. M. Shweshein et al. This is an open access article distributed under the Creative Commons Attribution License, which permits unrestricted use, distribution, and reproduction in any medium, provided the original work is properly cited.

\begin{abstract}
The lipophilicity of ten ruthenium(II)-arene complexes was assessed by reversed-phase thin-layer chromatography (RP-TLC) on octadecyl silica stationary phase. The binary solvent systems composed of water and acetonitrile were used as mobile phase in order to determine chromatographic descriptors for lipophilicity estimation. Octanol-water partition coefficient, $\log K_{\mathrm{OW}}$, of tested complexes was experimentally determined using twenty-eight standard solutes which were analyzed under the same chromatographic conditions as target substances. In addition, $a b$ initio density functional theory (DFT) computational approach was employed to calculate $\log K_{\mathrm{OW}}$ values from the differences in Gibbs' free solvation energies of the solute transfer from $n$-octanol to water. A good overall agreement between DFT calculated and experimentally determined $\log K_{\mathrm{OW}}$ values was established $\left(R^{2}=\right.$ $0.8024-0.9658)$.
\end{abstract}

\section{Introduction}

Apart from being important in material science and catalysis, metal ions and their complexes play a significant role in the vital functions of living organisms. Numerous applications of metal-based compounds as both therapeutic and diagnostic agents as well as mineral supplements were studied in scope of relatively young but rapidly developing research discipline named medicinal inorganic chemistry [1]. Metal complexes have a long history of use as medicines with cytostatic, antirheumatic, or anti-inflammatory properties. Further, they have been used in treatment of cardiac and many other diseases, while the anticancer activity of metal complexes, especially of cisplatin related drugs, is of the greatest importance [2].
In addition to the widely used platinum-based chemotherapeutic drugs such as cisplatin or carboplatin, numerous non-platinum-based compounds were investigated as anticancer agents $[1,2]$. Among them various ruthenium complexes attracted recent attention. Their anticancer activity, as well as clinical toxicity, is clearly distinct from platinum complexes [3-5]. Diversity of modes of action that involves both extra- and intracellular processes was achieved through interactions of ruthenium complexes with plasma proteins, extracellular matrix collagens, actins on the cell surface, regulatory enzymes in the plasma membranes or cytoplasm, and DNA in the cell nucleus [6]. In addition, some ruthenium complexes exhibit greater efficacy against cancer metastasis than against primary tumors by modulating adhesion, migration, invasion, proteolytic degradation of extracellular 
matrix, and new blood vessel formation. Over the last thirty years the interest for various ruthenium complexes, their synthesis and potential anticancer activity is constantly increasing, among them ruthenium(II)-arene compounds are being in the focus of research.

Our previous studies have been focused on syntheses, characterization, and cytotoxic activity of series of ruthenium(II)-arene compounds [7-9]. It has been shown that the presence of arene ligand is crucial. It is involved in several steps: it stabilizes the +2 ruthenium oxidation state, affects the cell uptake, influences the interactions with potential intracellular targets, and provides satisfactory lipophilicity needed to cross the cell membrane.

Since the lipophilicity is one of the major parameters affecting important biological processes that follow drug intake such as adsorption, passage through membranes, drug-receptor interactions, metabolism, and toxicity of molecules [10], we have decided to put an emphasis on the determination and estimation of lipophilicity of several ruthenium(II)-arene complexes in the scope of the present work.

Octanol-water partition coefficient $\left(\log K_{\mathrm{OW}}\right)$ is a widely accepted measure of lipophilicity and can be determined for various compounds including metal complexes in several ways. According to original "shake-flask" method, $K_{\mathrm{OW}}$ is defined as a concentration ratio of compound distributed between $n$-octanol and aqueous phase. However, this timeconsuming method with many experimental limitations has been replaced with chromatographic methods such as reversed-phase thin-layer chromatography (RP-TLC) [11], high-performance liquid chromatography (HPLC) in both, isocratic and gradient elution modes $[12,13]$, microemulsion electrokinetic chromatography (MEEKC) [14], and immobilized artificial membrane chromatography (IAM) [15]. Also, many software applications such as ALOGP, KOWIN, and CLOGP that incorporate different calculation approaches and algorithms have been frequently applied. However, these commonly used programs for $\log K_{\mathrm{OW}}$ estimation are not suitable in the case of complex compounds simply because the appropriate input of the central metal atom is usually missing, therefore resulting in poor predictions [16].

While considerable effort has been devoted to studying the $\log K_{\mathrm{OW}}$ of organic compounds, to the best of our knowledge, only few papers are dealing with the measure of $\log K_{\mathrm{OW}}$ of various complexes mostly by the means of the shake-flask method, HPLC in various reversed-phase modalities, and MEKC [16], probably because of the fact that complex compounds exhibit a very intricate behavior in liquid-liquid partitioning systems including HPLC that requires a delicate approach [17].

However, despite its numerous advantages and long history of successful application for lipophilicity determination of small organic molecules, modern high-performance thinlayer chromatography is rarely used for $\log K_{\text {OW }}$ determination of complex compounds, except for a few cases [18].

On the other hand, modern computational approach to the estimation of lipophilicity of metal complexes mostly relies on quantitative structure property relationship studies
(QSPR) that establish quantitative models based on experimentally determined $\log K_{\mathrm{OW}}$ data and $a b$ initio calculated molecular descriptors, such as the case with numerous platinum-based complexes $[19,20]$. However, complete, reliable, and comparable data which correlate the $\log K_{\mathrm{OW}}$ with relevant properties and molecular descriptors are needed. Molecular descriptors often reflect complex and multiple physical interactions, and model should include as many descriptors as possible, often leading to difficult and ambiguous interpretation of the results. Another approach based on $a b$-initio calculations is to estimate the free solvation energy change for the solute transfer from $n$-octanol to pure aqueous phase. Modern theoretical methods in quantum chemistry, such as density functional theory (DFT), possess great predictive power and in conjunction with continuum solvation models are proven to be very reliable in the determination of the free energy of solvation [21]. It is noteworthy to mention that advantages of DFT are particularly important for transition metal compounds [22, 23], although they are not always considered as innocent systems. Continuum solvation models are efficient tools for studying solvent effects on molecular structure, spectra, and energetics [2427] and have been used with success for determination of partition coefficients [28], pKa values [29], redox potentials [30], and so forth. Improvement of solvent models is active area in research in computational chemistry, and models are continually being improved and new versions and models frequently appear [31]. From a great variety of solvent models, in this work the universal model proposed by Marenich et al, based on Density (SMD) [32] is used due to the proven accuracy for first-principle calculation of solvation energies [33].

In the present work the attention was focused on the application of RP-TLC as a simple, fast, and reliable tool to determine $\log K_{\mathrm{OW}}$ values of ten ruthenium(II)-arene complexes with potential anticancer and antiproliferative activity. Moreover, there is a serious lack of information in the present literature about lipophilicity assessment of complex compounds by the means of TLC. In order to assure accuracy of the proposed methodology we have employed $a b$-initio DFT computational approach to calculate $\log K_{\mathrm{OW}}$ values from the differences in Gibbs' free solvation energies of the solute transfer from $n$-octanol to water and to compare estimated values with experimentally determined ones.

Being simple, fast and reliable RP-TLC provides retention data in the form of $R_{F}$ and corresponding $R_{M}$ values ((1) and (2)) that can be further used to derive several chromatographic descriptors for lipophilicity estimation: $R_{M}^{0}, b, C_{0}$, and PC1. Consider the following:

$$
R_{F}=\frac{x}{f} .
$$

Parameters $x$ and $f$ represent migration distance of the solute and solvent front, respectively. Consider the following:

$$
\begin{gathered}
R_{M}=\log \left(\frac{1}{R_{F}}-1\right), \\
R_{M}=R_{M}^{0}+b \varphi .
\end{gathered}
$$


The first parameter $\left(R_{M}^{0}\right)$ represents the intercept in the linear relation between retention of a solute, $R_{M}$, and volume fraction of organic-mobile-phase modifier, $\varphi$ (3). As a value extrapolated to $0 \% \mathrm{v} / \mathrm{v}$ of organic solvent it accounts for partitioning of a solute between pure water and the nonpolar stationary phase [34].

Slope, $b$, indicates the rate at which the solubility of the solute increases with changes in the mobile-phase composition and it is related to the specific hydrophobic surface area of a solute molecule [35]. Parameter $C_{0}$, introduced by Bieganowska et al. [36], represents the ratio of $R_{M}^{0}$ and $b$ and is considered as a concentration of organic modifier in the mobile phase for which the distribution of the solute between the two phases is equal.

The last descriptor, the first principal component, PC1, is derived from principal component analysis (PCA), that is, principal component regression (PCR), multivariate chemometric methods often applied on chromatographic data. It has been demonstrated that the scores of the principal components (usually the PC1 scores are sufficient) are better correlated with $\log K_{\text {OW }}$ since PCA combines all chromatographic data in one single feature [37].

\section{Experimental}

2.1. Materials. All reagents and solvents, including standard substances, were of analytical or HPLC purity grade. They are purchased from commercial suppliers: Aldrich (Milwaukee, WI, USA), Fluka (Buchs, Switzerland), and Merck (Darmstadt, Germany), and used as received. Ruthenium(II)arene complex compounds (Figure 1) were synthesized and characterized as already described $[7,8]$.

In the present work a set of 28 standard solutes, mainly mono- and polysubstituted phenols, aromatic carboxylic acids, ketones, amines, esters, and few polyaromatic hydrocarbons, of known $\log K_{\mathrm{OW}}$ values (Table 1) were chosen for calibration. Experimentally determined values of $\log K_{\mathrm{OW}}$ were taken from the KOWWIN software (EPI Suite, v. 4.11 US EPA). The set of standard compounds was compiled considering that hydrogen bonds, for both proton accepting and proton donating, as well as dipolar interactions, among the overall interactions that solutes exhibit in a chromatographic environment, should be present in a way as to ensure the ability of the final model to describe the behavior of a diverse set of compounds. The optimal range of $\log K_{\mathrm{OW}}$ values was considered broad enough to provide a reliable regression performance (from 1 to $5 \log K_{\text {OW }}$ units). Dissociation constants for ionogenic compounds were collected from several sources and presented along with the calculated degree of ionization $(\alpha)$ at $\mathrm{pH}=6$ in Table 1.

2.2. Chromatographic Procedure. For all chromatographic experiments solutions of standard substances, as well as of studied ruthenium complex compounds, were prepared by dissolving appropriate amount of substance in acetone in concentration of $0.1 \mathrm{mg} / \mathrm{mL}$. Commercially available octadecyl modified silica aluminum sheets (Art. number 5559, Merck, Darmstadt, Germany) were cut into $10 \times 10 \mathrm{~cm}$ plates.
TABLE 1: Calibration set of standard compounds with experimentally determined $\log K_{\mathrm{OW}}$ values.

\begin{tabular}{|c|c|c|c|c|}
\hline No. & Compound & $\log K_{\mathrm{OW}}$ & $\mathrm{p} K a$ & $\begin{array}{c}\alpha \text { Ionization } \\
\text { degree }(\%)\end{array}$ \\
\hline 1 & 1,2,3-Benzotriazole & 1.44 & 8.37 & 0.42 \\
\hline 2 & 4-Chlorobenzoic acid & 2.65 & 3.98 & 99.05 \\
\hline 3 & 2-Nitrobenzaldehyde & 1.74 & - & - \\
\hline 4 & 4-Bromoaniline ${ }^{*}$ & 2.26 & 3.86 & $99.28^{*}$ \\
\hline 5 & Phenol & 1.46 & 10.09 & 0.01 \\
\hline 6 & Benzophenone & 3.18 & - & - \\
\hline 7 & 3-Nitrobenzaldehyde & 1.46 & - & - \\
\hline 8 & 4-Aminobenzoic acid & 0.83 & 4.65 & 95.72 \\
\hline 9 & Phthalimide ${ }^{*}$ & 1.15 & & $100.00^{*}$ \\
\hline 10 & 1,4-Benzoquinone & 0.20 & - & - \\
\hline 11 & 4-Nitrophenol & 1.91 & 7.15 & 6.61 \\
\hline 12 & 3-Nitrophenol & 2.00 & 8.28 & 0.52 \\
\hline 13 & Benzyl alcohol & 1.10 & - & - \\
\hline 14 & Naphthylamine ${ }^{*}$ & 2.25 & 3.92 & $99.18^{*}$ \\
\hline 15 & 2-Naphthol & 2.85 & 9.67 & 0.02 \\
\hline 16 & 4-Fluoroaniline* & 1.15 & 4.70 & $95.23^{*}$ \\
\hline 17 & 1-Naphthol & 2.85 & 9.34 & 0.05 \\
\hline 18 & 4-Hydroxybenzaldehyde & 1.35 & - & - \\
\hline 19 & 3-Chloronitrobenzene & 2.46 & - & - \\
\hline 20 & 2,4-Dichlorophenol & 3.06 & 7.85 & 1.39 \\
\hline 21 & 4-Methylphenol & 1.94 & 10.09 & 0.01 \\
\hline 22 & 4-Chlorophenol & 2.39 & 9.38 & 0.04 \\
\hline 23 & Anthracene & 4.45 & - & - \\
\hline 24 & Acetophenone & 1.58 & - & - \\
\hline 25 & 2-Aminophenol & 0.62 & 9.44 & 0.04 \\
\hline 26 & 4-t-Butylphenol & 3.31 & 10.31 & 0.00 \\
\hline 27 & 1,3,5-Trihydroxybenzene & 0.16 & 9.40 & 0.04 \\
\hline 28 & 2,6-Dimethylphenol & 2.36 & 10.59 & 0.00 \\
\hline
\end{tabular}

${ }^{*}$ Calculated to the corresponding protonated conjugated acid.

The plates were manually spotted with approximate volume of $1.0 \mu \mathrm{L}$ of freshly prepared solutions, at $5 \mathrm{~mm}$ distance from each edge of the plate. Mixtures of acetonitrile and water were used as mobile phases, with organic component content increasing in the range of $30-60 \% \mathrm{v} / \mathrm{v}$, with increment of $5 \%$. Other organic modifiers such as methanol and acetone have been tested, but some of the ruthenium complexes were either moved through the system nonselectively or completely retained at the starting points. They are, therefore, discarded from the further study. The chromatograms were developed using horizontal developing chamber (CAMAG, Mutenz, Switzerland). The solvent migration distance was about $4.5 \mathrm{~cm}$. The plates were visually inspected under the UV light $(254 \mathrm{~nm})$ and each zone was clearly marked and its distance was manually measured. All measurements were done in triplicate and average values were used in further calculations. All experiments were performed at ambient temperature $\left(22 \pm 2^{\circ} \mathrm{C}\right)$. 


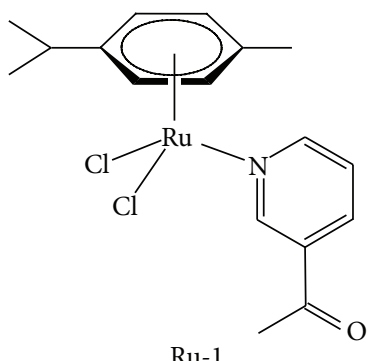

Ru-1

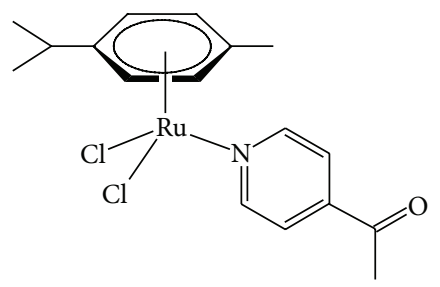

$\mathrm{Ru}-2$

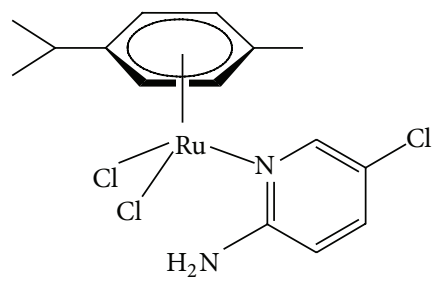

$\mathrm{Ru}-3$

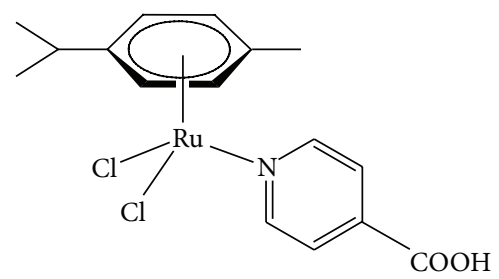

$\mathrm{Ru}-4$

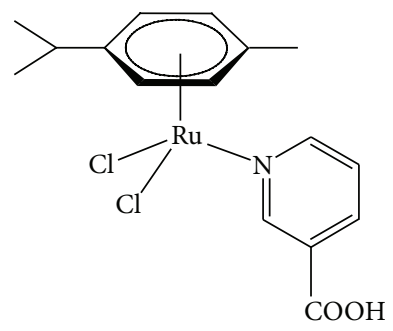

$\mathrm{Ru}-5$

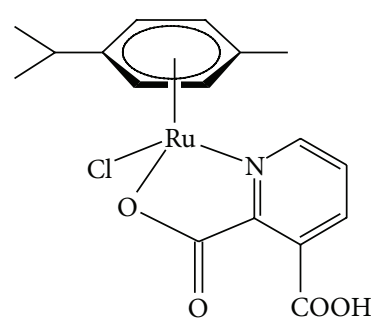

Ru-6

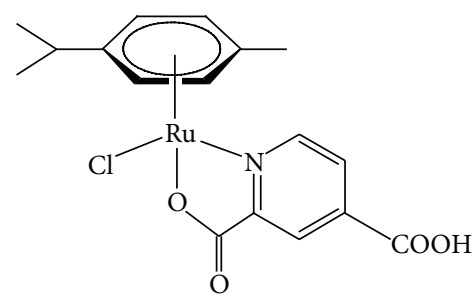

Ru-7

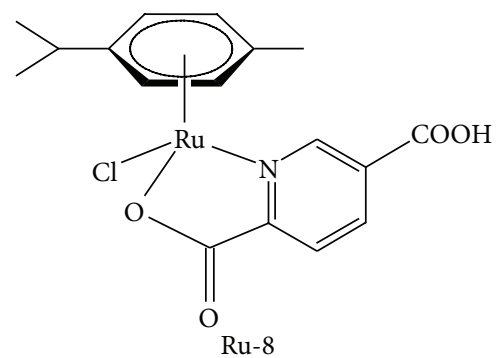

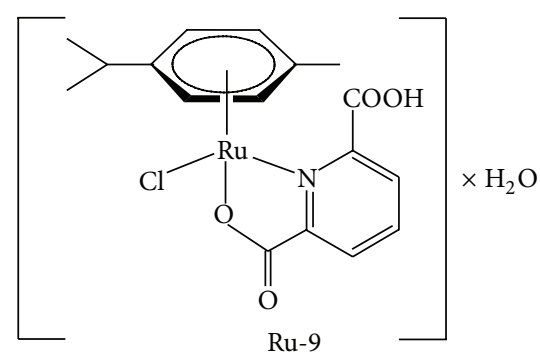<smiles>Cc1ccc(C(C)C)c(Cl)c1</smiles>

$\mathrm{Ru}-10$

Figure 1: Structures of studied ruthenium(II)-arene complexes.

2.3. Calculation of Chromatographic Descriptors. The following chromatographic descriptors: $R_{M}^{0}, b$, and $C_{0}$, were calculated based on the retention data (Table 2). $R_{M}^{0}$ and $b$ were obtained as the intercept and slope, respectively, according to (3). The hydrophobicity parameter $C_{0}$ was calculated as the ratio $\left(-R_{M}^{0} / b\right)$. However, this parameter did not show promising retention- $-\log K_{\mathrm{OW}}$ models, and was excluded from the further study. All necessary calculations 
TABLE 2: Retention of standard compounds and studied ruthenium complexes given as $R_{F}$ values.

\begin{tabular}{|c|c|c|c|c|c|c|c|c|}
\hline \multirow{2}{*}{ Number } & \multirow{2}{*}{ Compound } & \multicolumn{7}{|c|}{ Volume fraction of acetonitrile (\%) } \\
\hline & & 30 & 35 & 40 & 45 & 50 & 55 & 60 \\
\hline 1 & 1,2,3-Benzotriazole & - & - & 0.31 & 0.39 & 0.48 & 0.51 & 0.53 \\
\hline 2 & 4-Chlorobenzoic acid & - & - & 0.13 & 0.16 & 0.22 & 0.24 & 0.38 \\
\hline 3 & 2-Nitrobenzaldehyde & - & - & 0.11 & 0.14 & 0.21 & 0.24 & 0.30 \\
\hline 4 & 4-Bromoaniline & - & - & 0.06 & 0.08 & 0.15 & 0.18 & 0.25 \\
\hline 5 & Phenol & - & - & 0.26 & 0.33 & 0.40 & 0.50 & 0.57 \\
\hline 6 & Benzophenone & - & - & 0.03 & 0.05 & 0.07 & 0.09 & 0.15 \\
\hline 7 & 3-Nitrobenzaldehyde & - & - & 0.11 & 0.14 & 0.23 & 0.24 & 0.30 \\
\hline 8 & 4-Aminobenzoic acid & - & - & 0.44 & 0.48 & 0.54 & 0.56 & 0.60 \\
\hline 9 & Phthalimide & - & - & 0.24 & 0.30 & 0.41 & 0.43 & 0.48 \\
\hline 10 & 1,4-Benzoquinone & - & - & 0.40 & 0.43 & 0.49 & 0.50 & 0.52 \\
\hline 11 & 4-Nitrophenol & - & - & 0.24 & 0.28 & 0.33 & 0.46 & 0.52 \\
\hline 12 & 3-Nitrophenol & - & - & 0.16 & 0.20 & 0.27 & 0.41 & 0.49 \\
\hline 13 & Benzyl alcohol & - & - & 0.42 & 0.50 & 0.53 & 0.59 & 0.64 \\
\hline 14 & Naphthylamine & - & - & 0.12 & 0.16 & 0.22 & 0.33 & 0.41 \\
\hline 15 & 2-Naphthol & - & - & 0.13 & 0.18 & 0.27 & 0.37 & 0.43 \\
\hline 16 & 4-Fluoroaniline & - & - & 0.26 & 0.28 & 0.36 & 0.48 & 0.52 \\
\hline 17 & 1-Naphthol & - & - & 0.12 & 0.13 & 0.19 & 0.33 & 0.39 \\
\hline 18 & 4-Hydroxybenzaldehyde & - & - & 0.41 & 0.39 & 0.48 & 0.61 & 0.63 \\
\hline 19 & 3-Chloronitrobenzene & - & - & 0.07 & 0.09 & 0.11 & 0.22 & 0.28 \\
\hline 20 & 2,4-Dichlorophenol & - & - & 0.09 & 0.12 & 0.27 & 0.33 & 0.40 \\
\hline 21 & 4-Methylphenol & - & - & 0.26 & 0.30 & 0.36 & 0.48 & 0.54 \\
\hline 22 & 4-Chlorophenol & - & - & 0.17 & 0.24 & 0.30 & 0.39 & 0.51 \\
\hline 23 & Anthracene & - & - & 0.01 & 0.02 & 0.02 & 0.09 & 0.15 \\
\hline 24 & Acetophenone & - & - & 0.23 & 0.26 & 0.31 & 0.41 & 0.51 \\
\hline 25 & 2-Aminophenol & - & - & 0.42 & 0.42 & 0.51 & 0.62 & 0.66 \\
\hline 26 & 4-t-Butylphenol & - & - & 0.07 & 0.09 & 0.16 & 0.28 & 0.39 \\
\hline 27 & 1,3,5-Trihydroxybenzene & - & - & 0.73 & 0.67 & 0.77 & 0.80 & 0.84 \\
\hline 28 & 2,6-Dimethylphenol & - & - & 0.11 & 0.16 & 0.23 & 0.33 & 0.41 \\
\hline 29 & $\mathrm{Ru}-1$ & 0.01 & 0.01 & 0.02 & 0.04 & 0.06 & 0.11 & - \\
\hline 30 & $\mathrm{Ru}-2$ & 0.00 & 0.01 & 0.02 & 0.04 & 0.06 & 0.12 & - \\
\hline 31 & $\mathrm{Ru}-3$ & 0.01 & 0.01 & 0.02 & 0.04 & 0.06 & 0.15 & - \\
\hline 32 & $\mathrm{Ru}-4$ & 0.01 & 0.02 & 0.05 & 0.07 & 0.10 & 0.14 & - \\
\hline 33 & $\mathrm{Ru}-5$ & 0.01 & 0.03 & 0.06 & 0.08 & 0.13 & 0.16 & - \\
\hline 34 & $\mathrm{Ru}-6$ & 0.08 & 0.13 & 0.19 & 0.24 & 0.31 & 0.37 & - \\
\hline 35 & $\mathrm{Ru}-7$ & 0.23 & 0.28 & 0.32 & 0.37 & 0.44 & 0.49 & - \\
\hline 36 & $\mathrm{Ru}-8$ & 0.11 & 0.15 & 0.23 & 0.27 & 0.33 & 0.45 & - \\
\hline 37 & $\mathrm{Ru}-9$ & 0.05 & 0.07 & 0.13 & 0.16 & 0.21 & 0.29 & - \\
\hline 38 & $\mathrm{Ru}-10$ & 0.02 & 0.04 & 0.07 & 0.11 & 0.19 & 0.24 & - \\
\hline
\end{tabular}

were performed using the Data analysis tool-pack (Microsoft Excel 2010). The obtained models are summarized in Table 3 followed by accompanying statistics.

2.4. Modeling and Validation of TLC Retention- $\log K_{O W}$ Relationship. Calibration models based on individual $R_{M}$ values as well as extrapolated $R_{M}^{0}$ and $b$ descriptors were established using standard set of compounds (Table 1) and ordinary least squares procedure (Data analysis tool-pack, Microsoft Excel 2010). Principal component regression was employed instead of separate calculation of PC1 score values, using singular value decomposition (SVD) algorithm, followed by random subset cross-validation (five splits and one iteration) procedure. All calculations were made with PLS Tool Box v. 7.2., (Eigenvectors Research Inc.) for MATLAB 
TABLE 3: Linear relationship between solute retention $\left(R_{M}\right)$ and organic modifier volume fraction $(\varphi)$. Accompanied statistics $(R$ : Pearson's correlation coefficient, F: Fisher's parameter, S.D.: model standard deviation, $n$ : number of calibration points) with values of intercept $\left(R_{M}^{0}\right)$ and slope $(-b)$.

\begin{tabular}{|c|c|c|c|c|c|c|}
\hline \multirow{2}{*}{ Compound } & \multicolumn{6}{|c|}{ Chromatographic parameters } \\
\hline & $R_{M}^{0}$ & $-b$ & $R$ & S.D. & $F$ & $n$ \\
\hline 1,2,3-Benzotriazole & $1.13 \pm 0.16$ & $2.05 \pm 0.32$ & 0.9642 & 0.052 & 39.65 & 5 \\
\hline 4-Chlorobenzoic acid & $1.98 \pm 0.18$ & $2.83 \pm 0.36$ & 0.9770 & 0.056 & 62.92 & 5 \\
\hline 2-Nitrobenzaldehyde & $1.99 \pm 0.11$ & $2.73 \pm 0.22$ & 0.9901 & 0.035 & 149.91 & 5 \\
\hline 4-Bromoaniline & $2.74 \pm 0.19$ & $3.81 \pm 0.38$ & 0.9858 & 0.059 & 103.48 & 5 \\
\hline Phenol & $1.64 \pm 0.05$ & $2.94 \pm 0.11$ & 0.9981 & 0.017 & 768.73 & 5 \\
\hline Benzophenone & $2.86 \pm 0.11$ & $3.42 \pm 0.23$ & 0.9935 & 0.036 & 229.29 & 5 \\
\hline 3-Nitrobenzaldehyde & $1.98 \pm 0.20$ & $2.73 \pm 0.39$ & 0.9707 & 0.062 & 48.96 & 5 \\
\hline 4-Aminobenzoic acid & $0.65 \pm 0.07$ & $1.38 \pm 0.14$ & 0.9848 & 0.022 & 96.39 & 5 \\
\hline Phthalimide & $1.40 \pm 0.18$ & $2.32 \pm 0.35$ & 0.9671 & 0.056 & 43.29 & 5 \\
\hline 1,4-Benzoquinone & $0.61 \pm 0.08$ & $1.10 \pm 0.15$ & 0.9723 & 0.024 & 51.88 & 5 \\
\hline 4-Nitrophenol & $1.66 \pm 0.13$ & $2.83 \pm 0.25$ & 0.9886 & 0.039 & 129.19 & 5 \\
\hline 3-Nitrophenol & $2.22 \pm 0.15$ & $3.67 \pm 0.30$ & 0.9900 & 0.048 & 147.99 & 5 \\
\hline Benzyl alcohol & $0.87 \pm 0.07$ & $1.86 \pm 0.13$ & 0.9924 & 0.021 & 195.84 & 5 \\
\hline Naphthylamine & $2.40 \pm 0.08$ & $3.75 \pm 0.15$ & 0.9976 & 0.024 & 612.44 & 5 \\
\hline 2-Naphthol & $2.30 \pm 0.10$ & $3.70 \pm 0.21$ & 0.9954 & 0.033 & 323.64 & 5 \\
\hline 4-Fluoroaniline & $1.56 \pm 0.16$ & $2.68 \pm 0.32$ & 0.9789 & 0.051 & 68.73 & 5 \\
\hline 1-Naphthol & $2.44 \pm 0.23$ & $3.75 \pm 0.46$ & 0.9785 & 0.072 & 67.56 & 5 \\
\hline 4-Hydroxybenzaldehyde & $1.16 \pm 0.25$ & $2.33 \pm 0.49$ & 0.9396 & 0.078 & 22.59 & 5 \\
\hline 3-Chloronitrobenzene & $2.67 \pm 0.25$ & $3.75 \pm 0.50$ & 0.9747 & 0.078 & 57.07 & 5 \\
\hline 2,4-Dichlorophenol & $2.73 \pm 0.30$ & $4.35 \pm 0.60$ & 0.9728 & 0.095 & 52.89 & 5 \\
\hline 4-Methylphenol & $1.61 \pm 0.10$ & $2.80 \pm 0.194$ & 0.9928 & 0.031 & 207.53 & 5 \\
\hline 4-Chlorophenol & $2.10 \pm 0.08$ & $3.49 \pm 0.16$ & 0.9969 & 0.025 & 487.62 & 5 \\
\hline Anthracene & $4.37 \pm 0.50$ & $5.96 \pm 0.98$ & 0.9616 & 0.155 & 36.87 & 5 \\
\hline Acetophenone & $1.66 \pm 0.15$ & $2.75 \pm 0.29$ & 0.9836 & 0.046 & 89.03 & 5 \\
\hline 2-Aminophenol & $1.15 \pm 0.19$ & $2.37 \pm 0.37$ & 0.9657 & 0.059 & 41.45 & 5 \\
\hline 4-t-Butylphenol & $3.16 \pm 0.17$ & $4.93 \pm 0.35$ & 0.9927 & 0.055 & 204.43 & 5 \\
\hline 1,3,5-Trihydroxybenzene & $0.38 \pm 0.29$ & $1.79 \pm 0.57$ & 0.8754 & 0.090 & 9.84 & 5 \\
\hline 2,6-Dimethylphenol & $2.41 \pm 0.06$ & $3.77 \pm 0.13$ & 0.9984 & 0.020 & 908.70 & 5 \\
\hline $\mathrm{Ru}-1$ & $3.90 \pm 0.32$ & $5.45 \pm 0.74$ & 0.7903 & 0.155 & 6.65 & 6 \\
\hline $\mathrm{Ru}-2$ & $3.97 \pm 0.22$ & $5.71 \pm 0.51$ & 0.8797 & 0.106 & 13.69 & 6 \\
\hline $\mathrm{Ru}-3$ & $3.96 \pm 0.16$ & $5.75 \pm 0.38$ & 0.8263 & 0.079 & 8.61 & 6 \\
\hline $\mathrm{Ru}-4$ & $3.24 \pm 0.32$ & $4.56 \pm 0.74$ & 0.9351 & 0.154 & 27.86 & 6 \\
\hline $\mathrm{Ru}-5$ & $3.20 \pm 0.30$ & $4.69 \pm 0.69$ & 0.8732 & 0.145 & 12.84 & 6 \\
\hline $\mathrm{Ru}-6$ & $1.97 \pm 0.08$ & $3.24 \pm 0.18$ & 0.9564 & 0.037 & 42.93 & 6 \\
\hline $\mathrm{Ru}-7$ & $1.13 \pm 0.08$ & $2.02 \pm 0.19$ & 0.9875 & 0.039 & 157.10 & 6 \\
\hline $\mathrm{Ru}-8$ & $1.88 \pm 0.14$ & $3.22 \pm 0.31$ & 0.9563 & 0.066 & 42.81 & 6 \\
\hline Ru-9 & $2.35 \pm 0.17$ & $3.58 \pm 0.40$ & 0.9010 & 0.084 & 17.25 & 6 \\
\hline $\mathrm{Ru}-10$ & $3.04 \pm 0.14$ & $4.73 \pm 0.31$ & 0.9824 & 0.066 & 110.36 & 6 \\
\hline
\end{tabular}

R2011a (Mathworks Inc.). Quality of obtained models was assessed by the means of Pearson's $R^{2}$ and accompanying statistics.

2.5. DFT Computational Details. The common logarithm of $K_{\mathrm{OW}}$ is calculated as

$$
\log K_{\mathrm{OW}}=\frac{\Delta G_{\mathrm{sol}(\text { water })}-\Delta G_{\mathrm{sol}(\mathrm{oct})}}{2.303 R T}
$$

where $\Delta G_{\text {sol }}$ is the standard state solvation free energy of a given complex in octanol (oct) or in water (water) at $T=$ $298 \mathrm{~K}$. The standard-state solvation free energy is defined as the free energy of transfer from the gas phase to the condensed phase under standard state conditions. Because the gas-phase free energies are calculated with respect to a standard state of $1 \mathrm{~atm}$, a correction factor of $R T \ln (24.46)$ $\left(1.894 \mathrm{kcal} \mathrm{mol}^{-1}\right.$ at $\left.298 \mathrm{~K}\right)$ needs to be added to convert it into the standard state of $1 \mathrm{~mol} \mathrm{dm}^{-3}$. 
TABLE 4: Calibration models and accompanying statistics.

\begin{tabular}{lcll}
\hline $\begin{array}{l}\text { Model } \\
\text { number }\end{array}$ & Model type & Equation & Statistics \\
\hline 1 & OLS & $R_{M 40 \%}=(-0.17 \pm 0.09)+(0.44 \pm 0.04) \log K_{\mathrm{OW}}$ & $R^{2}=0.8067, \mathrm{~S} . \mathrm{D} .=0.22, P<10^{-4}, n=28$ \\
2 & OLS & $R_{M 45 \%}=(-0.17 \pm 0.08)+(0.38 \pm 0.04) \log K_{\mathrm{OW}}$ & $R^{2}=0.7882, \mathrm{~S} . \mathrm{D} .=0.20, P<10^{-4}, n=28$ \\
3 & OLS & $R_{M 50 \%}=(-0.32 \pm 0.09)+(0.37 \pm 0.04) \log K_{\mathrm{OW}}$ & $R^{2}=0.7666, \mathrm{~S} . \mathrm{D} .=0.20, P<10^{-4}, n=28$ \\
4 & OLS & $R_{M 55 \%}=(-0.34 \pm 0.09)+(0.29 \pm 0.04) \log K_{\mathrm{OW}}$ & $R^{2}=0.6521, \mathrm{~S} . \mathrm{D} .=0.15, P<10^{-4}, n=28$ \\
5 & OLS & $R_{M}^{0}=(0.3 \pm 0.2)+(0.83 \pm 0.06) \log K_{\mathrm{OW}}$ & $R^{2}=0.8857, \mathrm{~S} . \mathrm{D} .=0.33, P<10^{-4}, n=28$ \\
6 & OLS & $b=(1.2 \pm 0.2)+(0.98 \pm 0.08) \log K_{\mathrm{OW}}$ & $R^{2}=0.8388, \mathrm{~S} . \mathrm{D} .=0.31, P<10^{-4}, n=28$ \\
7 & PCR & $\log K_{\mathrm{OW}}=0.58 R_{M 40 \%}+0.63 R_{M 45 \%}+0.62 R_{M 50 \%}-0.95 R_{M 55 \%}$ & RMSEC $=0.375 ; R_{\mathrm{Cal}}^{2}=0.8502$ \\
& & & RMSECV $=0.418 ; R_{\mathrm{CV}}^{2}=0.8146$ \\
\hline
\end{tabular}

TABLE 5: Selected average bond lengths $(\AA)$ and valence angles $\left({ }^{\circ}\right)$ for available crystallographic data (a) and comparison with DFT energyminimized structures (b).

\begin{tabular}{|c|c|c|c|c|c|c|c|c|}
\hline \multirow{2}{*}{ Comp. } & \multicolumn{4}{|c|}{ Bond lengths $(\AA)$} & \multicolumn{4}{|c|}{ Valence angles $\left({ }^{\circ}\right)$} \\
\hline & $\mathrm{M}-\mathrm{C}$ & $\mathrm{M}-\mathrm{Cl}$ & $\mathrm{M}-\mathrm{N}$ & $\mathrm{M}-\mathrm{O}$ & $\mathrm{O}-\mathrm{M}-\mathrm{N}$ & $\mathrm{O}-\mathrm{M}-\mathrm{Cl}$ & $\mathrm{N}-\mathrm{M}-\mathrm{Cl}$ & $\mathrm{Cl}-\mathrm{M}-\mathrm{Cl}$ \\
\hline \multicolumn{9}{|l|}{$\mathrm{Ru}-1$} \\
\hline $\mathrm{a}$ & 2.18 & 2.40 & 2.13 & - & - & - & 86.0 & 87.0 \\
\hline $\mathrm{b}$ & 2.19 & 2.37 & 2.05 & - & - & - & 84.8 & 88.7 \\
\hline \multicolumn{9}{|l|}{$\mathrm{Ru}-7$} \\
\hline $\mathrm{a}$ & 2.18 & 2.41 & 2.10 & 2.10 & 77.9 & 86.7 & 85.3 & - \\
\hline $\mathrm{b}$ & 2.19 & 2.37 & 2.03 & 2.04 & 78.8 & 88.6 & 81.9 & - \\
\hline \multicolumn{9}{|l|}{$\mathrm{Ru}-9$} \\
\hline $\mathrm{a}$ & 2.19 & 2.40 & 2.16 & 2.09 & 76.9 & 83.7 & 85.2 & - \\
\hline $\mathrm{b}$ & 2.18 & 2.37 & 2.04 & 2.03 & 79.1 & 86.6 & 82.8 & - \\
\hline \multicolumn{9}{|l|}{$\mathrm{Ru}-10$} \\
\hline a & 2.19 & 2.42 & 2.10 & 2.09 & 78.0 & 85.2 & 85.2 & - \\
\hline $\mathrm{b}$ & 2.18 & 2.37 & 2.04 & 2.03 & 79.1 & 86.6 & 82.8 & - \\
\hline
\end{tabular}

All the DFT calculations have been carried out with the Gaussian 09, revision C.01 electronic structure program suite [38]. The local density approximation characterized by the Vosko-Wilk-Nusair (SVWN5) parametrization [39] has been used for the gas phase geometry optimizations, proven to be accurate for geometries of Werner type complexes [40-42]. The ruthenium cation was described by LANL2DZ relativistic effective core potentials (ECP) that replace 28 core electrons with a nonlocal effective potential and associated basis set for remaining electrons [43]. 6-31G+ $(\mathrm{d}, \mathrm{p})$ basis set [44] was used for all other atoms. For the determination of $\log K_{\mathrm{OW}}, \mathrm{M} 06 \mathrm{~L}$ density functional $[45,46]$, with the ECP LANL2TZ basis set [47] for ruthenium, and 6-311+G(2d,p) for other atoms were used. The free energies of solvation were calculated using continuum solvation model based on density (SMD) [32]. With SMD, the $298 \mathrm{~K}$ solvation Gibbs energy is defined as the difference between the solvent and gas electronic energies [48], necessitating corresponding gas-phase calculation.

\section{Results and Discussion}

For all studied compounds a good linearity between retention constants $\left(R_{M}\right)$ and the volume fraction of acetonitrile was obtained resulting in well-established chromatographic descriptors $\left(R_{M}^{0}\right.$ and $b$ ) (Table 3 ), even in the case of strongly polar or ionizable compounds as well as highly hydrophobic solutes. Although $C_{0}$ was initially calculated, this parameter was later excluded from further consideration, because of statistically significant, but poor, correlation with $\log K_{\mathrm{OW}}$ values $\left(R^{2}=0.4978, P<10^{-4}\right)$.

All calibration models were obtained using entire set of standard compounds. Accompanying statistics and equations are summarized in Table 4. Depending on the particular model, some of standard compounds were identified as outliers, with borderline statistical significance. However, all data were kept because of lack of reasonable justification for outlier removal. The best model was achieved using $R_{M}^{0}$ parameter $\left(R^{2}=0.8857\right)$, while direct calibration of retention in $55 \%$ acetonitrile-water system (model no. 4) showed the worst correlation $\left(R^{2}=0.6521\right)$. There is obvious deterioration in statistical performance of calibration models based on $R_{M}$ values with an increase of acetonitrile content in mobile phase; however, with exception of the model number 4, no model can be statistically justified as being preferred over the others. Use of direct calibration is more convenient 
TABLE 6: Chromatographically determined and computationally estimated $\log K_{\mathrm{OW}}$ values of studied ruthenium complexes.

\begin{tabular}{|c|c|c|c|c|c|c|c|c|c|c|}
\hline \multirow{3}{*}{ Comp. } & \multicolumn{7}{|c|}{ Chromatographically determined } & \multicolumn{3}{|c|}{ Estimated } \\
\hline & \multicolumn{7}{|c|}{ Model number } & \multicolumn{3}{|c|}{ Based on ab initio (DFT) computations } \\
\hline & 1 & 2 & 3 & 4 & 5 & 6 & 7 & $\begin{array}{l}\Delta G_{\text {sol }}(\text { oct }) \\
(\mathrm{kcal} / \mathrm{mol})\end{array}$ & $\begin{array}{c}\Delta G_{\text {sol }}(\text { water }) \\
(\mathrm{kcal} / \mathrm{mol})\end{array}$ & $\log K_{\mathrm{OW}}$ \\
\hline $\mathrm{Ru}-1$ & $4.26 \pm 0.37$ & $4.03 \pm 0.38$ & $4.19 \pm 0.42$ & $4.31 \pm 0.56$ & $4.31 \pm 0.28$ & $4.38 \pm 0.34$ & 3.72 & -13.85 & -19.81 & 4.36 \\
\hline $\mathrm{Ru}-2$ & $4.13 \pm 0.37$ & $3.91 \pm 0.37$ & $4.09 \pm 0.41$ & $4.13 \pm 0.55$ & $4.39 \pm 0.28$ & $4.63 \pm 0.35$ & 3.65 & -13.27 & -19.35 & 4.45 \\
\hline $\mathrm{Ru}-3$ & $4.13 \pm 0.37$ & $3.91 \pm 0.37$ & $4.09 \pm 0.41$ & $3.74 \pm 0.52$ & $4.38 \pm 0.28$ & $4.68 \pm 0.36$ & 3.97 & -9.99 & -17.63 & 5.59 \\
\hline $\mathrm{Ru}-4$ & $3.42 \pm 0.33$ & $3.43 \pm 0.35$ & $3.50 \pm 0.38$ & $3.86 \pm 0.52$ & $3.52 \pm 0.25$ & $3.47 \pm 0.30$ & 2.91 & -14.48 & -19.60 & 3.75 \\
\hline $\mathrm{Ru}-5$ & $3.18 \pm 0.32$ & $3.24 \pm 0.34$ & $3.08 \pm 0.36$ & $3.63 \pm 0.51$ & $3.47 \pm 0.24$ & $3.59 \pm 0.30$ & 2.63 & -14.99 & -19.97 & 3.65 \\
\hline $\mathrm{Ru}-6$ & $1.81 \pm 0.30$ & $1.72 \pm 0.32$ & $1.81 \pm 0.34$ & $1.99 \pm 0.45$ & $2.00 \pm 0.22$ & $2.11 \pm 0.27$ & 1.63 & -20.77 & -23.24 & 1.81 \\
\hline $\mathrm{Ru}-7$ & $1.15 \pm 0.31$ & $1.07 \pm 0.33$ & $1.15 \pm 0.35$ & $1.24 \pm 0.46$ & $0.98 \pm 0.23$ & $0.87 \pm 0.28$ & 1.14 & -20.51 & -22.57 & 1.50 \\
\hline $\mathrm{Ru}-8$ & $1.61 \pm 0.30$ & $1.59 \pm 0.32$ & $1.68 \pm 0.34$ & $1.49 \pm 0.45$ & $1.88 \pm 0.22$ & $2.09 \pm 0.27$ & 1.78 & -19.35 & -21.61 & 1.66 \\
\hline $\mathrm{Ru}-9$ & $2.33 \pm 0.30$ & $2.36 \pm 0.32$ & $2.39 \pm 0.34$ & $2.55 \pm 0.46$ & $2.44 \pm 0.22$ & $2.46 \pm 0.27$ & 2.14 & -16.88 & -19.70 & 2.06 \\
\hline $\mathrm{Ru}-10$ & $2.99 \pm 0.32$ & $2.80 \pm 0.33$ & $2.56 \pm 0.34$ & $2.87 \pm 0.47$ & $3.27 \pm 0.24$ & $3.63 \pm 0.31$ & 2.59 & -15.64 & -19.15 & 2.57 \\
\hline
\end{tabular}

compared to calculation of $R_{M}^{0}, b$, and PCR since it does not require additional experimental and computational work. However, $R_{M}^{0}$ and $b$ can be assessed for both highly lipophilic and polar compounds that exhibit measurable retention $\left(0.2<R_{F}<0.8\right)$ in different range of organic modifier volume ratios, in contrast to direct calibration based on isocratic chromatographic conditions. Because of its physical meaning, $R_{M}^{0}$ parameter might be the most suitable.

The calculated gas-phase geometries of all complexes under investigation are in excellent agreement with available X-ray crystal structures $[7,8]$ (Table 5).

Chromatographically determined $\log K_{\mathrm{OW}}$ values were obtained using retention data (Table 2) and established calibration models (Table 3). Data are summarized in Table 6. All estimation methods (models 1-7) give mutually coherent data, with no statistical difference in between. Also there is a good overall agreement between DFT calculated and experimentally determined values $\left(R^{2}=0.8024-0.9658\right)$.

Studied ruthenium(II)-arene complexes exhibit unusually high lipophilicity, in the range of 1-4 log units, compared with reported $\log K_{\mathrm{OW}}$ values of different series of platinum(II) and platinum(IV) complexes $[19,20]$ as well as with some Ru-( $\eta^{6}$-arene) compounds of similar structure that show significantly low octanol-water distribution coefficients [49]. However, all mentioned compounds have been positively charged, which might explain their low lipophilicity in comparison with neutral complexes investigated in current study.

\section{Conclusion}

The lipophilicity of ten ruthenium(II)-arene complexes with potential anticancer and antiproliferative activity has been determined by means of RPTLC on RP-18 silica as stationary and binary acetonitrile-water solvent systems as mobile phase. Based on retention data corresponding chromatographic descriptors for lipophilicity assessment have been obtained. Most of the experimental findings described above have been confirmed by DFT free energy calculations of complexes in octanol and water as solvents, using solvation model based on density (SMD). As results are promising, it can be considered as reliable tool for prediction of lipophilicity and rational design of coordination compounds with desired properties.

\section{Conflict of Interests}

The authors declare that they have no conflict of interests.

\section{Acknowledgment}

This work has been supported by the Ministry of Education, Science and Technological Development of Serbia, Grant no. 172017.

\section{References}

[1] T. Storr, K. H. Thompson, and C. Orvig, "Design of targeting ligands in medicinal inorganic chemistry," Chemical Society Reviews, vol. 35, no. 6, pp. 534-544, 2006.

[2] D. Gaynor and D. M. Griffith, "The prevalence of metal-based drugs as therapeutic or diagnostic agents: beyond platinum," Dalton Transactions, vol. 41, no. 43, pp. 13239-13257, 2012.

[3] E. S. Antonarakis and A. Emadi, "Ruthenium-based chemotherapeutics: are they ready for prime time?" Cancer Chemotherapy and Pharmacology, vol. 66, no. 1, pp. 1-9, 2010.

[4] M. Galanski, V. B. Arion, M. A. Jakupec, and B. K. Keppler, "Recent developments in the field of tumor-inhibiting metal complexes," Current Pharmaceutical Design, vol. 9, no. 25, pp. 2078-2089, 2003.

[5] A. Vacca, M. Bruno, A. Boccarelli et al., "Inhibition of endothelial cell functions and of angiogenesis by the metastasis inhibitor NAMI-A," British Journal of Cancer, vol. 86, no. 6, pp. 993-998, 2002.

[6] A. Levina, A. Mitra, and P. A. Lay, "Recent developments in ruthenium anticancer drugs," Metallomics, vol. 1, no. 6, pp. 458470, 2009. 
[7] S. Grgurić-Šipka, I. Ivanović, G. Rakić et al., "Ruthenium(II)arene complexes with functionalized pyridines: synthesis, characterization and cytotoxic activity," European Journal of Medicinal Chemistry, vol. 45, no. 3, pp. 1051-1058, 2010.

[8] I. Ivanović, S. Grgurić-Šipka, N. Gligorijević et al., "X-ray structure and cytotoxic activity of a picolinate ruthenium(II)arene complex," Journal of the Serbian Chemical Society, vol. 76, no. 1, pp. 53-61, 2011.

[9] N. Gligorijević, S. Aranđelović, L. Filipović et al., "Picolinate ruthenium(II)-arene complex with in vitro antiproliferative and antimetastatic properties: comparison to a series of ruthenium(II)-arene complexes with similar structure," Journal of Inorganic Biochemistry, vol. 108, pp. 53-61, 2012.

[10] J. A. Platts, S. P. Oldfield, M. M. Reif, A. Palmucci, E. Gabano, and D. Osella, "The RP-HPLC measurement and QSPR analysis of $\log$ Po/w values of several Pt(II) complexes," Journal of Inorganic Biochemistry, vol. 100, no. 7, pp. 1199-1207, 2006.

[11] A. Tsantili-Kakoulidou, "Lipophilicity: assessment by RP/TLC and HPLC," in Encyclopedia of Chromatography, vol. 2, pp. 1400-1407, Taylor \& Francis, Boca Raton, Fla, USA, 3rd edition, 2011.

[12] C. Giaginis and A. Tsantili-Kakoulidou, "Current state of the art in HPLC methodology for lipophilicity assessment of basic drugs. A review," Journal of Liquid Chromatography and Related Technologies, vol. 31, no. 1, pp. 79-96, 2008.

[13] K. Valkó, "Application of high-performance liquid chromatography based measurements of lipophilicity to model biological distribution," Journal of Chromatography A, vol. 1037, no. 1-2, pp. 299-310, 2004.

[14] C. W. Huie, "Recent applications of microemulsion electrokinetic chromatography," Electrophoresis, vol. 27, no. 1, pp. 60-75, 2006.

[15] C. Giaginis and A. Tsantili-Kakoulidou, "Alternative measures of lipophilicity: from octanol-water partitioning to IAM retention," Journal of Pharmaceutical Sciences, vol. 97, no. 8, pp. 29843004, 2008.

[16] L. S. Foteeva, D. A. Trofimov, O. V. Kuznetsova et al., "A quantitative structure-activity approach for lipophilicity estimation of antitumor complexes of different metals using microemulsion electrokinetic chromatography," Journal of Pharmaceutical and Biomedical Analysis, vol. 55, no. 3, pp. 409-413, 2011.

[17] É. A. Enyedy, D. Hollender, and T. Kiss, "Lipophilicity of kinetically labile metal complexes through the example of antidiabetic $\mathrm{Zn}(\mathrm{II})$ and VO(IV) compounds," Journal of Pharmaceutical and Biomedical Analysis, vol. 54, no. 5, pp. 1073-1081, 2011.

[18] M. Rusu, D. Rusu, and C. Sârbu, "Lipophilicity of metallic complexes of 4-methoxyphenyl-4' - chlorobenzoylhydrazine as estimated from principal component analysis of thin layer chromatographic retention data," Analytical Letters, vol. 32, no. 15, pp. 2999-3011, 1999.

[19] I. V. Tetko, I. Jaroszewicz, J. A. Platts, and J. Kuduk-Jaworska, "Calculation of lipophilicity for Pt(II) complexes: experimental comparison of several methods," Journal of Inorganic Biochemistry, vol. 102, no. 7, pp. 1424-1437, 2008.

[20] J. A. Platts, D. E. Hibbs, T. W. Hambley, and M. D. Hall, "Calculation of the hydrophobicity of platinum drugs," Journal of Medicinal Chemistry, vol. 44, no. 3, pp. 472-474, 2001.

[21] A. Jalan, R. W. Ashcraft, R. H. West, and W. H. Green, "Predicting solvation energies for kinetic modeling," Annual Reports on the Progress of Chemistry C, vol. 106, pp. 211-258, 2010.
[22] C. J. Cramer and D. G. Truhlar, "Density functional theory for transition metals and transition metal chemistry," Physical Chemistry Chemical Physics, vol. 11, no. 46, pp. 10757-10816, 2009.

[23] M. Reiher, "A theoretical challenge: Transition-metal compounds," Chimia, vol. 63, no. 3, pp. 140-145, 2009.

[24] C. J. Cramer and D. G. Truhlar, "Implicit solvation models: equilibria, structure, spectra, and dynamics," Chemical Reviews, vol. 99, no. 8, pp. 2161-2200, 1999.

[25] J. Tomasi, B. Mennucci, and R. Cammi, "Quantum mechanical continuum solvation models," Chemical Reviews, vol. 105, no. 8 , pp. 2999-3094, 2005.

[26] C. J. Cramer and D. G. Truhlar, "A universal approach to solvation modeling," Accounts of Chemical Research, vol. 41, no. 6, pp. 760-768, 2008.

[27] C. J. Cramer and D. G. Truhlar, "Reply to comment on: a universal approach to solvation modeling," Accounts of Chemical Research, vol. 42, no. 4, pp. 493-497, 2009.

[28] R. F. Ribeiro, A. V. Marenich, C. J. Cramer, and D. G. Truhlar, "The solvation, partitioning, hydrogen bonding, and dimerization of nucleotide bases: a multifaceted challenge for quantum chemistry," Physical Chemistry Chemical Physics, vol. 13, no. 23, pp. 10908-10922, 2011.

[29] C. C. R. Sutton, G. V. Franks, and G. da Silva, "First principles pKa calculations on carboxylic acids using the SMD solvation model: effect of thermodynamic cycle, model chemistry, and explicit solvent molecules," The Journal of Physical Chemistry B, vol. 116, pp. 11999-12006, 2012.

[30] P. Jaque, A. V. Marenich, C. J. Cramer, and D. G. Truhlar, "Computational electrochemistry: the aqueous $\mathrm{Ru}^{3+} \mid \mathrm{Ru}^{2+}$ reduction potential," Journal of Physical Chemistry C, vol. 111, no. 15, pp. 5783-5799, 2007.

[31] A. V. Marenich, C. J. Cramer, and D. G. Truhlar, "Generalized born solvation model SM12," Journal of Chemical Theory and Computation, vol. 9, no. 1, pp. 609-620, 2013.

[32] A. V. Marenich, C. J. Cramer, and D. G. Truhlar, "Universal solvation model based on solute electron density and on a continuum model of the solvent defined by the bulk dielectric constant and atomic surface tensions," Journal of Physical Chemistry B, vol. 113, no. 18, pp. 6378-6396, 2009.

[33] M. Koláŕ, J. Fanfrlık, M. Lepšık, F. Forti, F. J. Luque, and P. Hobza, "Assessing the accuracy and performance of implicit solvent models for drug molecules: conformational ensemble approaches," The Journal of Physical Chemistry B, vol. 117, pp. 5950-5962, 2013.

[34] C. Horváth, W. Melander, and I. Molnár, "Solvophobic interactions in liquid chromatography with nonpolar stationary phases," Journal of Chromatography A, vol. 125, no. 1, pp. 129156, 1976.

[35] F. Murakami, "Retention behaviour of benzene derivatives on bonded reversed-phase columns," Journal of Chromatography A, vol. 178, no. 2, pp. 393-399, 1979.

[36] M. L. Bieganowska, A. Doraczynska-Szopa, and A. Petruczynik, "The retention behavior of some sulfonamides on different TLC plates. 2. Comparison of the selectivity of the systems and quantitative determination of hydrophobicity parameters," Journal of Planar Chromatography Modern TLC, vol. 8, no. 2, pp. 122-128, 1995.

[37] C. Sârbu, K. Kuhajda, and S. Kevresan, "Evaluation of the lipophilicity of bile acids and their derivatives by thin-layer chromatography and principal component analysis," Journal of Chromatography A, vol. 917, no. 1-2, pp. 361-366, 2001. 
[38] M. J. Frisch, G. W. Trucks, H. B. Schlegel et al., Gaussian 09, Revision A.1, Gaussian, Wallingford, Conn, USA, 2009.

[39] S. Vosko, L. Wilk, and M. Nusair, "Accurate spin-dependent electron liquid correlation energies for local spin density calculations: a critical analysis," Canadian Journal of Physics, vol. 58, pp. 1200-1211, 1980.

[40] T. K. Kundu, R. Bruyndonckx, C. Daul, and P. T. Manoharan, "A density functional approach to the jahn-teller effect of $\left[\mathrm{Cu}(\mathrm{en})_{3}\right]^{2+}$ as a model for a macrobicyclic cage complex of copper(II)," Inorganic Chemistry, vol. 38, no. 17, pp. 3931-3934, 1999.

[41] M. R. Bray, R. J. Deeth, V. J. Paget, and P. D. Sheen, “The relative performance of the local density approximation and gradientcorrected density functional theory for computing metal-ligand distances in Werner-type and organometallic complexes," International Journal of Quantum Chemistry, vol. 61, no. 1, pp. 85-91, 1996.

[42] M. Zlatar, M. Gruden-Pavlović, M. Güell, and M. Swart, "Computational study of the spin-state energies and UV-Vis spectra of bis(1,4,7-triazacyclononane) complexes of some firstrow transition metal cations," Physical Chemistry Chemical Physics, vol. 15, pp. 6631-6639, 2013.

[43] P. J. Hay and W. R. Wadt, "Ab initio effective core potentials for molecular calculations. Potentials for the transition metal atoms Sc to Hg," The Journal of Chemical Physics, vol. 82, no. 1, pp. 270283, 1985.

[44] P. C. Hariharan and J. A. Pople, "The influence of polarization functions on molecular orbital hydrogenation energies," Theoretica Chimica Acta, vol. 28, no. 3, pp. 213-222, 1973.

[45] Y. Zhao and D. G. Truhlar, "A new local density functional for main-group thermochemistry, transition metal bonding, thermochemical kinetics, and noncovalent interactions," Journal of Chemical Physics, vol. 125, no. 19, Article ID 194101, 2006.

[46] Y. Zhao and D. G. Truhlar, "The M06 suite of density functionals for main group thermochemistry, thermochemical kinetics, noncovalent interactions, excited states, and transition elements: Two new functionals and systematic testing of four M06-class functionals and 12 other functionals," Theoretical Chemistry Accounts, vol. 120, no. 1-3, pp. 215-241, 2008.

[47] L. E. Roy, P. J. Hay, and R. L. Martin, "Revised basis sets for the LANL effective core potentials," Journal of Chemical Theory and Computation, vol. 4, no. 7, pp. 1029-1031, 2008.

[48] R. F. Ribeiro, A. V. Marenich, C. J. Cramer, and D. G. Truhlar, "Use of solution-phase vibrational frequencies in continuum models for the free energy of solvation," Journal of Physical Chemistry B, vol. 115, no. 49, pp. 14556-14562, 2011.

[49] A. Martínez, C. S. K. Rajapakse, D. Jalloh, C. Dautriche, and R. A. Sánchez-Delgado, "The antimalarial activity of Ruchloroquine complexes against resistant Plasmodium falciparum is related to lipophilicity, basicity, and heme aggregation inhibition ability near water/n-octanol interfaces," Journal of Biological Inorganic Chemistry, vol. 14, no. 6, pp. 863-871, 2009. 

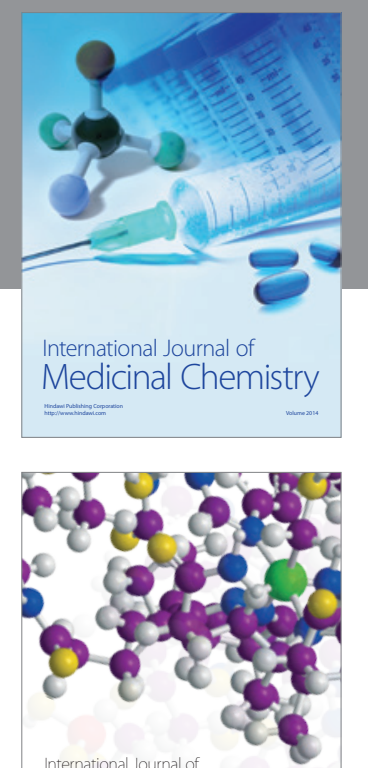

\section{Carbohydrate} Chemistry

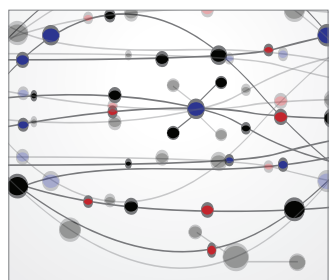

The Scientific World Journal
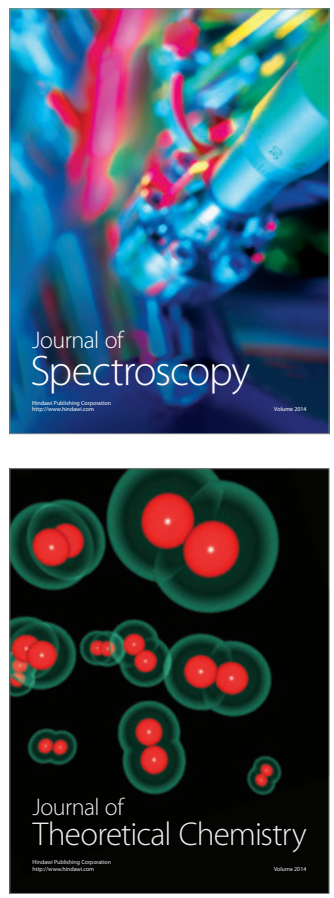
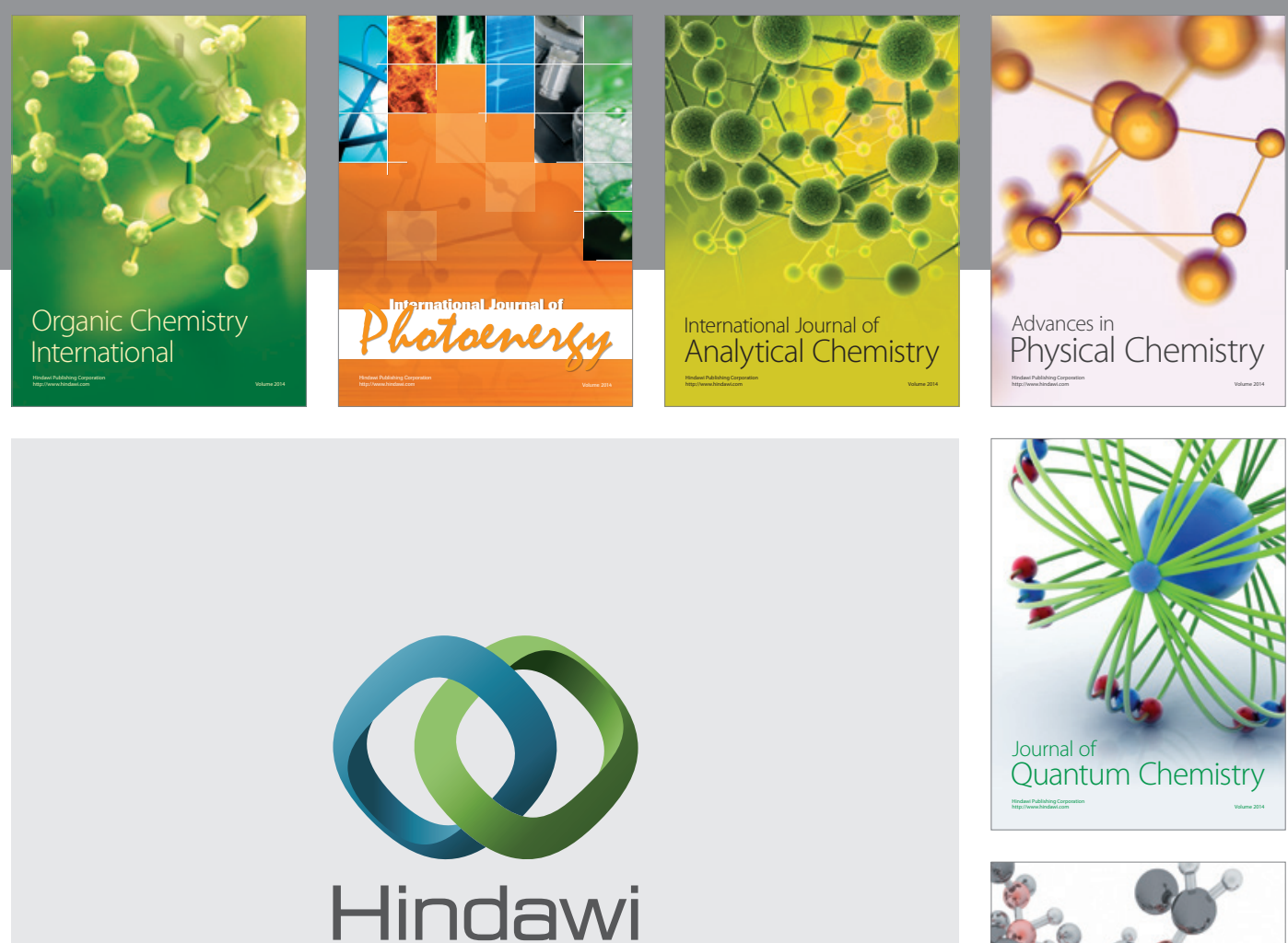

Submit your manuscripts at

http://www.hindawi.com

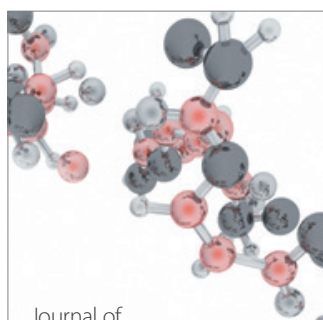

Analytical Methods

in Chemistry

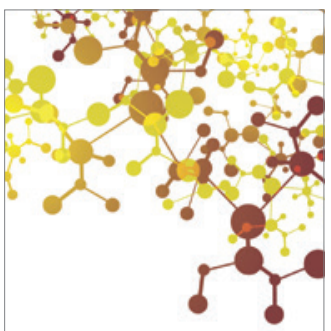

Journal of

Applied Chemistry

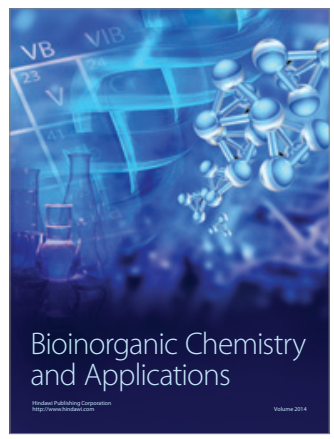

Inorganic Chemistry
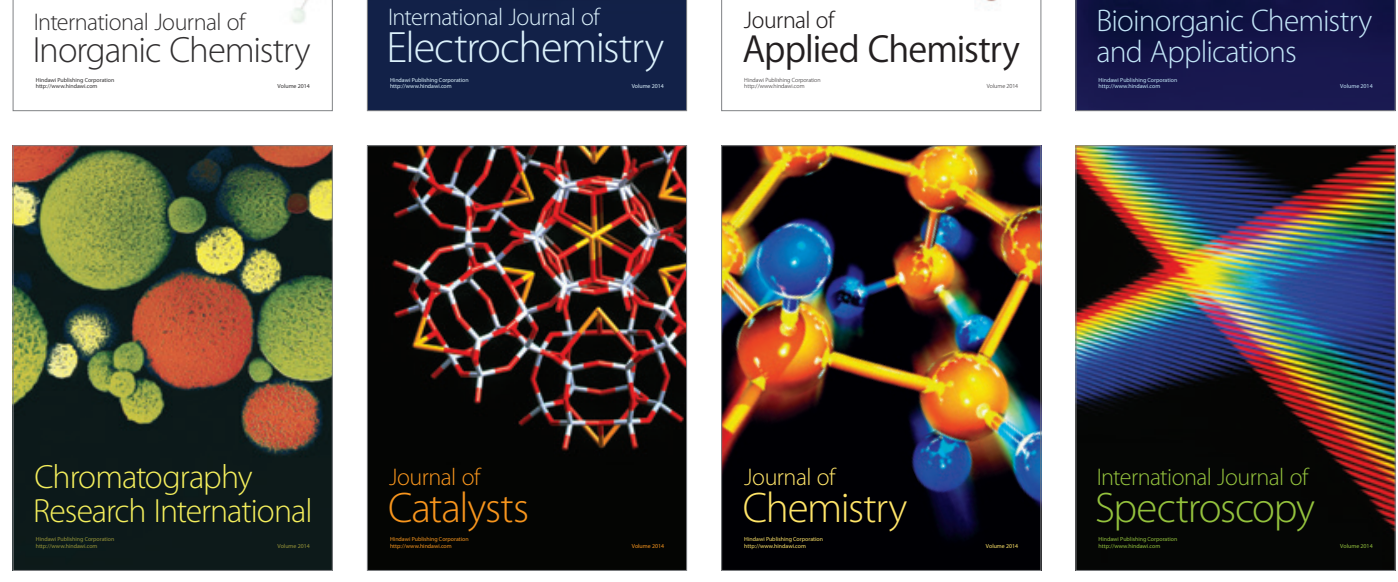